\title{
Modal Logic Investigations in the Semantics of Counts-as
}

\author{
Davide Grossi, John-Jules Ch. Meyer,Frank Dignum \\ Utrecht University, \\ The Netherlands \\ \{davide,jj,dignum\}@cs.uu.nl
}

\begin{abstract}
The work investigates the logic underlying the representation of the non-regulative component of normative systems, the so-called counts-as. The analytic thesis we hold here is to view counts-as statements as statements which yield classifications and which hold only with respect to a context. These two aspects of the semantics of counts-as -the classificatory flavor, and the contextual character- are then investigated by means of modal logic techniques from a semantics-driven perspective, and a formalization of counts-as statements is thus proposed. The result is then compared in detail with previous work on the topic, and related with work which, despite developed in different areas of applied and philosophical logic, shares interesting technical and theoretical similarities with our proposal.
\end{abstract}

\section{Introduction}

In the study of the normative character of social reality, it is a widely acknowledged opinion that normative systems of high complexity, for example legal systems or institutional ones, consist of regulative as well as non-regulative components $([1,25,24,39,7])$. That is, they do not only regulate existing forms of behavior, but they actually specify and create new such forms.

Where the rule is purely regulative, behaviour which is in accordance with the rule could be given the same description or specification (the same answer to the question "What did he do?") whether or not the rule existed, provided the description or specification makes no explicit reference to the rule. But where the rule (or systems of rules) is constitutive, behaviour which is in accordance with the rule can receive specifications or descriptions which it could not receive if the rules did not exist [p.35] ([38]).

In legal theory the non-regulative component has been labeled in ways that emphasize a classificatory, as opposed to a normative/regulative, character: de- 
terminative rules ([41]), conceptual rules ([8]), qualification norms $([33])$, definitional norms ([24]). This characteristic of the non-regulative component of normative systems appears to be intermingled with a second, also widely acknowledged feature, namely the constitutive and conventional character of these components that have also been therefore called constitutive rules or constitutive norms ([36, 39]). In this view, statements to the effect that $\mathrm{X}$ counts as $\mathrm{Y}$ establish that the occurrence of $\mathrm{X}$ constitutes, in the sense of being a sufficient condition for, the occurrence of $\mathrm{Y}$. Alternative readings which can be found in the literature are: X conventionally generates $\mathrm{Y}([15])$, or $\mathrm{Y}$ supervenes on $\mathrm{X}$ $([20])$.

However, this "constitution" (or "generation", or "supervenience") is not absolute. Being conventional, it only holds within the specific context (institution, or normative system) in which that relation of constitution is effective, it is contextual. This feature has been particularly emphasized in [39], where constitutive rules are viewed as representable via the following type of statements: "X counts as $\mathrm{Y}$ in context C".

The aim of the present work is to build on the formal analysis of contextual classifications we have developed in [19] in order to provide a modal logic characterization of contextual classificatory statements, thus providing a formalization of a precise analytical option in the understanding of counts-as. To this aim, an important step will also be the detailed comparison of our proposal with the characterization of counts-as developed by Jones and Sergot in [26], in order to clearly display what kind of new insights our perspective enables.

The work is structured according to the following outline. In Section 2 the analytical thesis underpinning the formal analysis is presented and motivated. In Section 3 the logical machinery is introduced, discussed in detail, and by means of it a formalization of counts-as statements is proposed. In Section 4 our proposal is related with work theoretically and technically analogous to ours which has been developed in the area of epistemic logic and deontic logic. Our formal analysis of counts-as is then also used in order to provide an original counts-as based understanding of reduction approaches to deontic logic. In Section 5 the work is put in perspective with the formal characterization of counts-as proposed in [26]. Finally, in Section 6, issues concerning our proposal are discussed, and conclusions are drawn.

\section{Analytical Background}

In this section we expose the theoretical options presupposed by this work, distilling the basic ideas presented in [19]. We refer to that work for a more rigorous and detailed exposition of what follows.

\subsection{Counts-as as subsumption}

The way we ground a formal characterization of the classificatory view of countsas statements is extremely plain: if counts-as statements yield classifications, 
this means that they function as conceptual subsumption relations, that is, counts-as statements assert just that a concept $X$ is a subconcept of a concept $Y$. This analysis is inspired by the consideration of some well known problems of underspecification, or more technically open-texture $([22])$, typical of legal terminologies. We quote an excerpt from [21] neatly exposing this type of problems.

[Suppose a] legal rule forbids you to take a vehicle into the public park. Plainly this forbids an automobile, but what about bicycles, roller skates, toy automobiles? What about airplanes? Are these, as we say, to be called "vehicles" for the purpose of the rule or not? If we are to communicate with each other at all, and if, as in the most elementary form of law, we are to express our intentions that a certain type of behavior be regulated by rules, then the general words we use like "vehicle" in the case I consider must have some standard instance in which no doubts are felt about its application. There must be a core of settled meaning, but there will be, as well, a penumbra of debatable cases in which words are neither obviously applicable nor obviously ruled out. [...] We may call the problems which arise outside the hard core of standard instances or settled meaning "problems of the penumbra"; they are always with us whether in relation to such trivial things as the regulation of the use of the public park or in relation to the multidimensional generalities of a constitution.

Such "problems of the penumbra" can be indeed rephrased in terms of questions of the form what counts as what: what does count as a vehicle? The fact that bicycles count as vehicles would then be read as: "instances of the concept bicycle are always instances of the concept vehicle". In [19], these assertions are represented as mere conceptual subsumptions, i.e., expressions of the kind bicycle $\sqsubseteq$ vehicle. The semantics of these expressions is elementary. Given a domain $D$ of entities, and an interpretation function $\mathcal{I}$ of the concepts, a model $m=\langle D, \mathcal{I}\rangle$ satisfies the expression bicycle $\sqsubseteq$ vehicle iff $\mathcal{I}$ (bicycle) $\subseteq$ $\mathcal{I}(\text { vehicle })^{1}$. The classificatory aspect of counts-as can be therefore formally captured by conceptual subsumptions.

Via such statements, normative systems can establish the ontology they use in order to distribute obligations, rights, prohibitions, permissions. Vehicles are not admitted in public parks (general norm), but then, if bicycles count as vehicles (classification), bicycles are not admitted in public parks (specific norm). This interplay phenomenon between regulative and non-regulative components, between norms and classificatory statements, has been discussed in various literature reports $([37,7,17])$. Our claim is that this interplay works on a classificatory (i.e., ontological) basis, and that counts-as is nothing but the basic brick of these classifications. Each normative system incorporates a classification (i.e., ontology) providing an interpretation of the concepts with

\footnotetext{
${ }^{1}$ This is nothing but a clause of the semantics of TBoxes in description logics ([4]).
} 
which it is specified on the domain of entities it is supposed to regulate.

\subsection{Counts-as as contextual subsumption}

In the previous section we focused on the classificatory aspect of counts-as. What about the contextual one? In the presence of multiple incompatible countsas statements concerning a same concept "problems of the penumbra" arise. The concept of vehicle has a "penumbra" because it can get multiple interpretations which subsume or do not subsume other concepts such as bicycle or skateboard. In fact, a same thing can in some cases count as a vehicle, and in some others not: in what context does which of the competing counts-as statements hold? In particular, different normative systems have different non-regulative components: what counts as a vehicle in one public park regulation might well not count as a vehicle in another public park regulation. Counts-as statements will therefore be analyzed as subsumption statements which do not hold unconditionally, but only with respect to a specific normative system or, in general, with respect to a specific context.

This is clarified elaborating on the simple example introduced above. Consider two normative systems $a$ and $b$ regulating the traffic within public parks in two different municipalities. According to (in the context of) the public parks regulation of the first municipality bicycles do not count as vehicles, according to (in the context of) the public parks regulation of the second one bicycles do count as vehicles. In both $a$ and $b$ cars count as vehicles. The non-regulative part of these systems might be formally specified on a language $\mathcal{L}_{\text {traffic }}$ containing at least the following atomic concepts: vehicle, bicycle, car. A formal specification of the different classifications holding in the two systems $a$ and $b$ should then be able to express that the subsumption of the concept bicycle under the concept vehicle holds in the context of the first municipality, but not in the context of the second one, and that the subsumption of the concept car under the concept vehicle holds in both contexts. Put formally:

$M_{a} \vDash$ bicycle $\sqsubseteq$ vehicle;

$M_{b} \not \models$ bicycle $\sqsubseteq$ vehicle;

$M_{a} \vDash$ car $\sqsubseteq$ vehicle;

$M_{b} \vDash$ car $\sqsubseteq$ vehicle;

where $M_{a}$ and $M_{b}$ are sets of models $m$ for $\mathcal{L}_{\text {traffic }}$. In fact, contexts are formalized as sets of models, i.e. the set of interpretations of the concepts presupposed by those normative systems ${ }^{2}$. Intuitively, the idea is then to view the context of a counts-as statement precisely as the set of relevant interpretations of the concepts used to specify the normative system to which the statement belongs.

To sum up, our analytical option consists in viewing counts-as statements as conceptual subsumptions which are made true by a specific set of interpretations of the relevant concepts. How can this thesis be modeled within a modal logic setting? And would this translation provide new insights?

${ }^{2}$ For a detailed discussion of this conception of the notion of context we refer again to [19]. 


\section{Counts-as and Possible Worlds}

In this section we show how to use the previous intuitions in order to ground a possible world semantics for counts-as conditionals of the type investigated in [26], widely relying on well-known modal logic terminology and results exposed for instance in $[14,6]$.

\subsection{Logical preliminaries}

We will first introduce the languages we are going to work with: propositional n-modal languages $\mathcal{M L}_{n}([14])$. The alphabet of $\mathcal{M} \mathcal{L}_{n}$ contains: a finite set $\mathbb{P}$ of propositional atoms $p$; the set of boolean connectives $\{\neg, \wedge, \vee, \rightarrow\}$; two sets of modal operators $\left\{\square_{i}\right\}_{1 \leq i \leq n}$ and $\left\{\diamond_{i}\right\}_{1 \leq i \leq n}$. The set of well formed formulas $\phi$ of $\mathcal{M} \mathcal{L}_{n}$ is then defined by the following $\mathrm{BNF}$ :

$$
\phi::=p|\neg \phi| \phi_{1} \wedge \phi_{2}\left|\phi_{1} \vee \phi_{2}\right| \phi_{1} \rightarrow \phi_{2}\left|\square_{i} \phi\right| \diamond_{i} \phi .
$$

where $1 \leq i \leq n$. We will refer to formulas $\phi$ in which at least one modal operator occurs as modalized formulas.

Semantics for these languages is given via structures $\mathcal{M}=\langle\mathcal{F}, \mathcal{I}\rangle$, where:

- $\mathcal{F}$ is a n-frame, i.e., a structure $\left\langle W,\left\{R_{i}\right\}_{1 \leq i \leq n}\right\rangle$, where $W$ is the set of states (possible worlds) and $\left\{R_{i}\right\}_{1<i<n}$ is a family of $n$ accessibility relations $^{3}$. We will refer to the set of accessible worlds from a world $w$ via a relation $R_{i}$ as $r_{i}(w)$.

- $\mathcal{I}$ is an evluation function $\mathcal{F}: \mathbb{P} \longrightarrow \mathcal{P}(W)$ associating to each atom the set of states which make it true.

Satisfaction for these languages is then defined as follows:

$$
\begin{array}{rll}
\mathcal{M}, w \vDash p & \text { iff } & w \in \mathcal{I}(p) \\
\mathcal{M}, w \vDash \neg \phi_{1} & \text { iff } & \mathcal{M}, w \nvdash \phi_{1} \\
\mathcal{M}, w \vDash \phi_{1} \wedge \phi_{2} & \text { iff } & \mathcal{M}, w \vDash \phi_{1} \text { AND } \mathcal{M}, w \vDash \phi_{2} \\
\mathcal{M}, w \vDash \phi_{1} \vee \phi_{2} & \text { iff } & \mathcal{M}, w \vDash \phi_{1} \text { OR } \mathcal{M}, w \vDash \phi_{2} \\
\mathcal{M}, w \vDash \phi_{1} \rightarrow \phi_{2} & \text { iff } & \mathcal{M}, w \vDash \phi_{1} \text { IMPLIES } \mathcal{M}, w \vDash \phi_{2} \\
\mathcal{M}, w \vDash \square_{i} \phi & \text { iff } & \forall w^{\prime} \in r_{i}(w): \mathcal{M}, w^{\prime} \vDash \phi \\
\mathcal{M}, w \vDash \diamond_{i} \phi & \text { iff } & \exists w^{\prime} \in r_{i}(w): \mathcal{M}, w^{\prime} \vDash \phi .
\end{array}
$$

A formula $\phi$ is said to be valid in a model $\mathcal{M}$, in symbols $\mathcal{M} \vDash \phi$, iff for all $w$ in $W, \mathcal{M}, w \vDash \phi$. It is said to be valid in a frame $\mathcal{F}(\mathcal{F} \vDash \phi)$ if it is valid in all models based on that frame. Finally, it is said to be valid on a class of frames $\mathrm{F}(\mathrm{F} \vDash \phi)$ if it is valid in every frame $\mathcal{F}$ in $\mathrm{F}$.

\footnotetext{
$[14]$.

${ }^{3}$ The notation "n-frame" to indicate a frame with $n$ accessibility relations is taken from
} 


\subsection{Classifications and modal logic}

The first intuition we exposed in Section 2 consisted in regarding counts-as statements as subsumptions: "all instances of bicycle are also instances of vehicle". The obvious candidate for representing, or rather sketching, conceptual subsumption in propositional logic is material implication. Analogously, a subsumption considered to hold unconditionally would be formalized on a $\mathcal{M L}_{1}$ language by means of a strict implication for a S5 logic: $\square$ (bicycle $\rightarrow$ vehicle). The classificatory part of each normative system, considered in isolation, can then be modeled via structures for S5 systems. The point is what happens if we want to consider, under the same modal formalism, a variety of such structures, that is to say, if we want to represent many classificatory parts belonging to different normative system specifications. Such a step is necessary to handle the second claim we held in Section 2, i.e., the contextual nature of counts-as statements. Technically we are interested in a logic that can "locally" behave like an S5 logic but that can "globally" behave in a weaker way allowing for different (thus possibly inconsistent) classificatory representations at the same time.

In other words, we should find a multi-modal logic enabling as many modalities as the to be represented contexts, and retaining for these modalities as many characteristics of S5 as possible, but at the same time allowing for the satisfiability of expressions such as: $\square_{i}($ bicycle $\rightarrow$ vehicle $) \wedge \neg \square_{j}($ bicycle $\rightarrow$ vehicle).

A class of frames characterized by $\mathbf{S 5}$ logic is the class UNIV of universal frames, i.e., the class of frames s.t. for all $\forall w_{1}, w_{2}: w_{1} R_{i} w_{2}$. The question is then what kind of n-frames can meet our requirements ${ }^{4}$.

\subsection{Frames with contexts}

The candidate can be found in a quite well investigated class of n-frames ([31, 30,18]), that is, the class CXT of n-frames such that:

- CXT is serial, i.e., for all $R_{i} \in\left\{R_{i}\right\}_{1 \leq i \leq n}, \forall w, r_{i}(w) \neq \emptyset$;

- CXT is locally universal $l^{5}$, i.e., for all $R_{i} \in\left\{R_{i}\right\}_{1 \leq i \leq n}$, if $\forall w R_{i}$ is universal on $r_{i}(w)$ and $\forall w_{1}, w_{2}, r_{i}\left(w_{1}\right)=r_{i}\left(w_{2}\right)$, that is to say, that every world has access via $R_{i}$ to the same set of worlds, which we denote as $W_{i}$, and $R_{i}$ is also universal on that set.

${ }^{4}$ A quite natural way of answering our question might be to consider the class of $n$-frames in which each $R_{i}$ with $1 \leq i \leq n$ is an equivalence relation considering therefore clustered models for S5. However, this approach, which is proposed with different but related purposes in [27], leaves the interaction between contexts completely unconstrained in the sense that the various equivalence relations remain unrelated which is, in our perspective, an undesirable feature. In fact, we think of truth in a context as independent of the context from which it can be evaluated. In other words, the fact that a classification holds with respect to a context is actually context-independent. This feature will become evident in the semantics and in the axiomatization exposed, respectively, in Section 3.3 and in Section 3.4.

${ }^{5}$ In $[30]$ the term secondarily universal is used. 
Out of technicalities, these properties for $\left\{R_{i}\right\}_{1<i<n}$ cluster the domain of the frame in non-empty sets of worlds, one for each accessibility relation and define these accessibility relations in such a way that the set of accessible worlds corresponds, for each world in $W$, to the cluster. Yet an easier way to express this is to say that these $\mathrm{n}$-frames define, via $\left\{R_{i}\right\}_{1 \leq i \leq n}$, $\mathrm{n}$ consistent contexts, i.e., $\mathrm{n}$ non-empty sets of worlds. Noticeably, this class of frames implements in a straightforward way the thesis developed in context modeling according to which contexts can be soundly represented as sets of possible worlds $([40])$. It may also be instructive to notice that these clusters can overlap. In fact, being sets of worlds, they can be ordered via set theoretic inclusion and the usual set theoretic operations can be defined on them ${ }^{6}$. Because of all these features, the class CXT appears to be the natural candidate for translating, into a modal logic setting, the notion of context as set of (monadic first-order) models sketched in Section 2.

Models for a $\mathcal{M L}_{n}$ can be built on CxT n-frames in the obvious way. The satisfaction relation, then, results in the following.

Definition 1 (Satisfaction based on CXT n-frames)

Let $m$ be a model built on a CXT $n$-frame.

$$
\begin{array}{ccl}
\mathcal{M}, w \vDash \square_{i} \phi & \text { iff } \quad \forall w^{\prime} \in W_{i}: \mathcal{M}, w^{\prime} \vDash \phi \\
\mathcal{M}, w \vDash \nabla_{i} \phi & \text { iff } \quad \exists w^{\prime} \in W_{i}: \mathcal{M}, w^{\prime} \vDash \phi .
\end{array}
$$

The obvious clauses for non-modal formulas are omitted.

As it can be easily seen, the essential characteristic of CXT n-frames, which marks the difference with UNIV frames, consists in the fact that they do not make the $\mathrm{T}$ scheme $(\square \phi \rightarrow \phi)$ valid. This is indeed what one would first of all expect from a formalization of a notion of contextuality or locality via modal operators: if something holds in a context, it does not necessarily hold in general. On the other hand, $\mathrm{T}$ remains valid in a sort of contextualized formulation. In fact, formulas $\square_{i}\left(\square_{i} \phi \rightarrow \phi\right)$ are valid, that is to say, $\mathrm{T}$ schema remains contextually valid.

It may be worth noticing that, given the set of states $W$, it is always possible to define a specific CXT n-frame with $n=2^{W}$, that is to say, the $\mathrm{CXT}_{\top} \mathrm{C}$ frame containing all possible consistent contexts: $\left\langle W, \mathcal{P}^{+}(W)\right\rangle$. Consequently, each CXT can be viewed as a structure $\langle W, C\rangle$ where $C \subseteq \mathcal{P}^{+}(W)$.

\subsection{A multi-modal logic for contextual classifications}

We have thus a class of frames which models the conception of context underlying our analysis. To get to the logic of contextual implication at which we are aiming, the following has to be proved.

1. Consider a model $\mathcal{M}_{1}$ based on UNIV for $\mathcal{M} \mathcal{L}_{1}$, i.e., a model for $\mathbf{S 5}$, making a set of modalized formulas $\Phi$ on $\mathcal{M L}_{1}$ true. There always exists

\footnotetext{
${ }^{6}$ We did this in [19].
} 
a model $\mathcal{M}_{0}$ based on $\mathrm{CxT}$ for $\mathcal{M} \mathcal{L}_{1}$ such that it is always possible to define a sub-model $\mathcal{M}_{1}^{*}$ of $\mathcal{M}_{0}$ which makes exactly $\Phi$ true. Out of technicalities, this means that the classificatory part of a normative system, considered in isolation, can be represented as the set of $\square$-formulas which are true in a $\mathcal{M}_{0}$ model (Proposition 1)

2. Consider a family $\left\{\mathcal{M}_{i}\right\}_{1 \leq i \leq n}$ of $\mathbf{S 5}$ models for $\mathcal{M} \mathcal{L}_{1}$ built on UNIV frames. There always exists a model $\mathcal{M}_{0}$ based on CxT for $\mathcal{M} \mathcal{L}_{1}$ such that $\mathcal{M}_{i}=\phi$ iff $\mathcal{M}_{0} \models \phi^{\prime}$, where $\phi$ is modalized and $\phi^{\prime}$ is obtained from $\phi$ uniformly substituting occurrences of $\square$ and $\diamond$ with occurrences of $\square_{i}$ and $\diamond_{i}$. This is just the multi-modal extension of the previous result: the classificatory part of each of $\mathrm{n}$ different normative systems can be represented as the set of $\square_{i}$-formulas (with $1 \leq i \leq n$ ) which are true in a $\mathcal{M}_{0}$ model (Proposition 2).

3. The $\mathbf{K D} 4 \mathbf{5}_{\mathbf{n}}^{\mathbf{i}-\mathbf{j}}$ logic is sound and complete with respect to the family of frames underlying the aforementioned models. This logic, which has been investigated in $[32,31,30]$, is then the candidate to provide a logical characterization of the notion of counts-as exposed in Section 2 (Proposition $3)$.

Proposition 1 (From UNIV to CXT models)

Consider the model $\mathcal{M}_{1}$ for $\mathcal{M L}_{1}$ s.t. $\mathcal{M}_{1}=\left\langle W_{1}, R_{1}, \mathcal{I}_{1}\right\rangle$ with $R_{1}$ universal on $W_{1}$. There always exists a model $\mathcal{M}_{0}$ for $\mathcal{M L}_{1}$ s.t. $\mathcal{M}_{0}=\left\langle W_{0}, R_{0}, \mathcal{I}_{0}\right\rangle$, where: $R_{0}$ is serial and locally universal; and there exists a non empty subset $W_{1}^{*}$ of $W_{0}$ such that if $\mathcal{M}_{1}^{*}=\left\langle W_{1}^{*}, R_{0} \cap W_{1}^{*} \times W_{1}^{*}, \mathcal{I}_{1}^{*}\left\lceil W_{1}^{*}\right\rangle\right.$ with $R^{*} \cap W_{1}^{*} \times W_{1}^{*}$ universal, then $\mathcal{M}_{1} \models \phi$ iff $\mathcal{M}_{1}^{*} \models \phi$ iff $\mathcal{M}^{*} \models \phi$, where $\phi$ is a modalized formula.

Proof. To prove the proposition we show that it is always possible to construct the desired structure. Consider a model $\mathcal{M}_{1}^{*}=\left\langle W_{1}^{*}, R_{1}^{*}\right.$, $\left.\mathcal{I}_{1}^{*}\right\rangle$ s.t. $\mathcal{M}_{1}^{*}$ is isomorphic to $\mathcal{M}_{1}$. Isomorphism trivially yields modal invariance, therefore: $\mathcal{M}_{1} \models \phi$ iff $\mathcal{M}_{0} * \models \phi$ (obviously, $R_{1}^{*}$ is universal). Model $\mathcal{M}_{1}^{*}$ can then be expanded to the structure $\mathcal{M}^{*}=\left\langle W_{0}, R_{0}, \mathcal{I}_{0}\right\rangle$ where: $W_{0} \supseteq W_{1}^{*} ; R_{1}^{*}=R_{0} \cap$ $W_{1}^{*} \times W_{1}^{*}$ and $\forall w \in W_{0}, r_{0}(w)=W_{1}^{*} ; \mathcal{I}_{1}^{*}=\mathcal{I}_{0}\left\lceil W_{1}^{*}\right.$. The obtained structure $\mathcal{M}_{0}$ is, by construction, serial and locally universal.

Proposition 2 (From many UNIV models to one CXT model) Consider now a set of models $\mathcal{M}_{1}, \ldots, \mathcal{M}_{n}$ for $\mathcal{M L}_{1}$ on UNIV frames. Then there always exists a model $\mathcal{M}_{0}$ for $\mathcal{M L}_{n}$ s.t. $\mathcal{M}_{0}=\left\langle W_{0},\left\{R_{i}^{*}\right\}_{1 \leq i \leq n}, \mathcal{I}_{0}\right\rangle$ on a CXT $n$-frame with $W_{0} \supseteq \bigcup_{1 \leq i \leq n} W_{i}^{*}$, and $\mathcal{M}_{i}=\phi$ iff $\mathcal{M}_{0} \models \phi^{\prime}$, where $\phi$ is modalized and $\phi^{\prime}$ is obtained from $\phi$ uniformally substituting occurrences of $\square$ and $\diamond$ with occurrences of $\square_{i}$ and $\diamond_{i}$.

Proof. Follows directly from Proposition 1.

Proposition 1 and 2 prove that serial and locally universal frames are the structures we are looking for. The natural question is now: is there a logic 
characterizing the class of these structures? The answer is positive and the system at issue corresponds to a quite widely investigated logic: $\mathbf{K D}_{\mathbf{n}}^{\mathbf{i}-\mathbf{j}}$ ([32, $31,30]$ ).

Logic $\mathbf{K D 4 5} \mathbf{i}-\mathbf{j}$ is axiomatized via the following axioms and rules schemata:

$(P) \quad$ all tautologies of propositional calculus

$(K) \quad \square_{i}\left(\phi_{1} \rightarrow \phi_{2}\right) \rightarrow\left(\square_{i} \phi_{1} \rightarrow \square_{i} \phi_{2}\right)$

(D) $\quad \neg \square_{i} \perp$

$\left(4^{i-j}\right) \quad \square_{i} \phi \rightarrow \square_{j} \square_{i} \phi$

$\left(5^{i-j}\right) \quad \neg \square_{i} \phi \rightarrow \square_{j} \neg \square_{i} \phi$

$(M P) \quad \phi_{1}, \phi_{1} \rightarrow \phi_{2} / \phi_{2}$

$(N) \quad \phi / \square_{i} \phi$

The system is a multi-modal homogeneous KD45 with the two interaction axioms $4^{i-j}$ and $5^{i-j 7}$.

Proposition 3 (Soundness and completeness)

Logic $\mathbf{K D 4 5} \mathbf{n}_{\mathbf{n}}^{\mathbf{i}-\mathbf{j}}$ is sound and complete with respect to the family of CXT $n$ frames.

Proof. Full proofs are worked out in $[31,30]$. Here we will provide just the sketch of a proof. The desired result can be obtained in two steps. First, via the canonical model, it can be proved that logic $\mathbf{K D} \mathbf{4 5} \mathbf{\mathbf { n }} \mathbf{\mathbf { i }} \mathbf{j}$ is complete with respect to the class of serial, transitive, and i-j euclidean $\left(w R_{i} w^{\prime} \wedge w R_{j} w^{\prime \prime} \rightarrow w^{\prime} R_{j} w^{\prime \prime}\right)$ frames $^{8}$. Soundness is, as usual, straightforward. Second, it can be proved that if $\mathcal{F}$ is a CXT n-frame then it is serial, transitive and $i-j$ euclidean. The reverse can be proved with respect to the class of serial, transitive and $\mathrm{i}-\mathrm{j}$ euclidean frames which are also rooted $\left(\exists w_{0} \in W\right.$ s.t. $\left.W=\left\{w \in W \mid w_{0} R_{i} w\right\}\right)$. The following well known invariance result about generated submodels ${ }^{9}$ can then be exploited to prove soundness and completeness also with respect to CxT nframes: if an n-modal logic is determined by (i.e., sound and complete w.r.t.) a class of $n$-frames, then it is determined by the class of its rooted frames ([14]).

At this stage the logical machinery is semantically and syntactically worked out and it can be therefore put at work ${ }^{10}$.

\footnotetext{
${ }^{7}$ Instead of $4^{i-j}$, it would be sufficient to assume a simple 4 axiom: $\square_{i} \phi \rightarrow \square_{i} \square_{i} \phi$ (see [30]).

${ }^{8}$ In [32], frames with this property are called hyper-euclidean.

${ }^{9}$ For the notion of generated submodel we refer the reader to [6].

${ }^{10}$ It is worth noticing, in passing, that model checking techniques for system $\mathbf{K D} \mathbf{4} \mathbf{5}_{\mathbf{n}}^{\mathbf{i}-\mathbf{j}}$ are available and are exposed in [35].
} 


\subsection{Contextual subsumption and multi-modal strict im- plication}

Using a multi-modal logic $\mathbf{K D} \mathbf{4 5} \mathbf{n}_{\mathbf{n}}^{\mathbf{i} \mathbf{j}}$ on a language $\mathcal{M L}_{n}$, counts-as statements can be formalized as follows.

Definition 2 (Counts-as formalized)

" $\phi_{1}$ counts as $\phi_{2}$ in context $i$ " is formalized in a multi-modal language $\mathcal{M L}_{n}$ as the strict implication $\square_{i}\left(\phi_{1} \rightarrow \phi_{2}\right)$ of logic $\mathbf{K D} 45_{\mathbf{n}}^{\mathbf{i}-\mathbf{j}}$.

It is instructive to make a few remarks. Two are the relevant features of this formal characterization. First of all, material implication between propositions is used as embodiment of the notion of classification, or subsumption. Second, this implication becomes strict in relation with the specific $\mathbf{K D} \mathbf{4 5} \mathbf{n}_{\mathbf{n}}^{\mathbf{i}-\mathbf{j}}$ modality. This modality implements, in a straightforward way, the thesis developed in context modeling according to which contexts can be soundly represented as sets of possible worlds ([40]). In particular, schemata $4^{i-j}$ and $5^{i-j}$ play a key role. What is their intuitive reading in the light of Definition 2 ? In fact, what the two schemata do, consists in making the nesting of the operators reducible which, leaving technicalities aside, means that truth and falsehood in contexts are somehow absolute. From the point of view of a theory of counts-as they express the fact that normative systems determine only the classifications holding for themselves and have no relevance in evaluating whether other classifications hold in other normative systems: the fact that bicycles count as vehicles in municipality $a$ is not something that municipality $b$ can deny ${ }^{11}$. To summarize, the truth of a counts-as statements is context independent. Besides, the $\mathbf{K D}_{\mathbf{4}} \mathbf{\mathbf { i }} \mathbf{\mathbf { j }}$ modality readily enables the possibility of dealing with inconsistent counts-as statements belonging to different normative system specifications: formulas such as $\square_{i}\left(\phi_{1} \rightarrow \phi_{2}\right) \wedge \neg \square_{j}\left(\phi_{1} \rightarrow \phi_{2}\right)$ are satisfiable. Finally, it is worth noticing that this modality appears to be also a valid candidate to represent the Searlean notion of institutional fact ${ }^{12}$ in a modal logic setting.

\section{Counts-As, Epistemic and Ideal States}

In this section we point to work in epistemic and deontic logic showing how the framework just exposed interestingly relates to issues that go beyond the analysis of social and institutional realities alone. This will also give some insights on how the aspects we highlighted in analyzing counts-as are actually of a very general nature.

\footnotetext{
${ }^{11}$ There are nevertheless forms of interaction between normative systems such as the "inheritance" of classifications between contexts which are in a subsystem/supersystem relation. Notice though that, since contexts are here viewed as sets of worlds, this situation can be easily modeled in our framework via formulas such as: $\square_{i} \phi \rightarrow \square_{j} \phi$ where $i$ subsumes $j$.

${ }^{12}$ On the notions of institutional fact and brute fact see [39].
} 


\subsection{Contexts and epistemic states}

Readers acquainted with epistemic logic have probably noticed striking similarities of the logic presented with logics usually used to represent epistemic states of different agents. In fact, logic $\mathbf{K D} \mathbf{4 5} \mathbf{5}_{\mathbf{n}}^{\mathbf{i}-\mathbf{j}}$ is a conservative extension of logic $\mathbf{K D 4 5}_{\mathbf{n}}$, the multi-modal version of logic KD45, which can be used to represent, in a basic way, the beliefs of a number of agents ([23]). The essen-

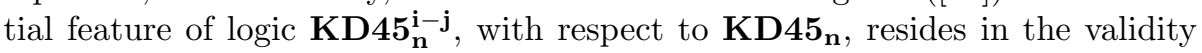
of interaction schemata $4^{i-j}$ and $5^{i-j}$ (See the axiomatization given in Section 3.4) which would mean, from a doxastic point of view, that the beliefs of agents are always the same no matters by which agent they are considered. This is obviously a quite unrealistic assumption since agent $j$ can believe that agent $i$ believes $\phi$, while indeed it does not. In some sense, the notion of context used here is too strong to model a notion of context as doxastic state of a given agent $^{13}$.

Nevertheless, a convincing epistemic interpretation of logic $\mathbf{K D 4 5} \mathbf{i} \mathbf{i} \mathbf{j}$ can be found once the notion of context is not interpreted as the epistemic state of one agent in a group of agents, but rather as one of the epistemic states one single agent can assume. What we called here context can therefore be doxastically interpreted as 'set of hypothesis', or 'set of presumable beliefs with respect to a given situation', or 'context of a given theory' etc.:

One may think of a researcher in physics who may consider an electron alternatively as a particle or as a wave, depending on whether s/he thinks classically or quantum-physically ([31]).

More precisely, formulas $\square_{i} \phi$ would intuitively mean: "the agent believes $\phi$ with respect to context (or situation, or opinion, or body of hypothesis) $i$ ". This perspective underpins various work carried out in the area of epistemic logic, especially when the core issue is the innocuous representation of inconsistencies $^{14}$. This is the case, for instance, of the model theoretic analysis of local reasoning proposed in [13] which is in many respects very similar to the semantics we presented in Section 3.4. The same logical framework proposed in the present paper, with a small difference, has instead been developed in [31] in order to provide a monotonic epistemic account of defeasible reasoning ${ }^{15}$. In that work, a modal operator is used in order to express what is believed according to one of the possible multiple defeasible extensions of the belief base of the agent $^{16}$ : $\square_{i} \phi$ means that $\phi$ holds in extension $i$, or also that the agent presumes $\phi$ within $i$. Noticeably, the semantics chosen for that operator is the same one

\footnotetext{
${ }^{13}$ In fact, much literature in context logics, like [10, 9], aims at formalizing weaker notions of contexts which can receive a sound epistemic reading.

${ }^{14} \mathrm{As}$ it has been stressed in Section 3.5, the possibility of representing conflicting classifications holding in different normative systems is also one of the motivating features of the logic presented here.

${ }^{15}$ The obtained logic is called EDL (epistemic default logic). See, for details, Chapter 4 of [31].

${ }^{16}$ The notion of extension, i.e., what can be defeasibly inferred from a body of knowledge, is one of the basic concepts of non-monotonic logic.
} 
based on CXT n-frames which we adopted here, apart for the fact that the seriality condition for $\left\{R_{i}\right\}_{1 \leq i \leq n}$ is dropped in order to be able to represent empty extensions, that is, inconsistent ways in which the agent can choose to extend its belief base. In the present paper instead, the inconsistent context, i.e., the empty set of world, is not admitted in the semantics, somehow presupposing the coherency of the classificatory part of normative systems.

\subsection{A counts-as reduction of deontic logic}

Deontic logic formalizes reasoning about "the distinction between what ideally is the case on the one hand, and what actually is the case on the other" ([24]). The standard deontic logic is the modal system KD in which $\square \phi$ and $\diamond \phi$ formulas are interpreted as, respectively, $\phi$ is obligatory (i.e. it holds in all "ideal" worlds) and $\phi$ is permitted (i.e. it holds in some "ideal" worlds). A plethora of alternative approaches to deontic notions is available in the literature ${ }^{17}$. In this section, we will concisely focus on those approaches which make use of the well-known reduction strategy first presented by Anderson in [2,3], and relate them with the notion of counts-as studied here.

The reduction strategy is based on the intuition according to which $\phi$ is ideally the case means that $\neg \phi$ "necessarily" implies a violation (of the relevant set of norms or deontic constraints), in symbols: $\square(\neg \phi \rightarrow V)$, where $V$ is a specific atom for which it is valid that $\diamond \neg V$, i.e., that the violation is not "necessary". The nature of the reduction lies in how this reference to a "necessity" is formally modeled. In the original proposal of Anderson the system chosen for the reduction was $\mathbf{K T}$. Here we consider alternative reductions based instead on system S5 such as the ones studied in $[12,27,30]$. Interpreting the $\square$ operator occurring in the reduction expression as a $\mathbf{S 5}$ necessity yields expressions formally identical to the one discussed at the beginning of our analysis in Section 3.2, i.e., S5 strict implications. In such settings, therefore, formulas $\square(\neg \phi \rightarrow V)$ could be rephrased as: the negation of $\phi$ counts unconditionally as a violation. According to this approach, deontic notions are then reduced to a kind of universal classification statements.

Taking this reduction strategy as a starting point, our analysis of counts-as statements as contextual classifications is then readily applicable and delivers a straightforward and intuitive way of treating contextual forms of obligations via a reduction based on $\mathbf{K D} \mathbf{4 5} \mathbf{n}_{\mathbf{n}}^{\mathbf{i}-\mathbf{j}}$ logic. The fact that $\phi$ is obligatory in context $i$ can be formalized as $\square_{i}(\neg \phi \rightarrow V)$ and read as: the negation of $\phi$ counts as a violation in context $i$. It becomes thus possible to express that $\phi$ is obligatory in context $i$ while $\neg \phi$ is permitted in context $j: \square_{i}(\neg \phi \rightarrow V) \wedge \diamond_{j}(\neg \phi \wedge \neg V)$. In this view, contextual obligations express just different ways of understanding the same notion of violation, and the problem whether something is obligatory or not in a given context amounts then - to use the terminology of Hart ${ }^{18}$ - to "a problem of the penumbra" of the concept of violation.

\footnotetext{
${ }^{17}$ See [28] for recent contributions in this field.
}

${ }^{18}$ See Section 2. 


\subsection{Deontics as a form of contextuality}

Interestingly and curiously, system $\mathbf{K D} \mathbf{4 5} \mathbf{5}_{\mathbf{n}}^{\mathbf{i}-\mathbf{j}}$ is used also in $[29,30]$ in order to express deontic constraints directed to agents. The fact that $\phi$ is obligatory for agent $i$ is expressed via formulas such as $\square_{i} \phi$, that is, $\operatorname{logic} \mathbf{K D} \mathbf{4} \mathbf{5}_{\mathbf{n}}^{\mathbf{i}-\mathbf{j}}$ is directly used as a form of deontic logic without any reduction strategy. Indexes refer, in that case, to agents and not to contexts as in our case, and the system is then intended as a multi-agent deontic logic. Nevertheless, this conception of obligations presupposes the understanding of the set of ideal states for some agent as a context in the sense used in the present work, and more precisely, as a context in which nothing happens to be classified as a violation, a kind of "ideal context".

With respect to this, the interesting point consists in understanding the technical and theoretical difference of representing deontic notions making use of system $\mathbf{K D} \mathbf{4 5} \mathbf{5}_{\mathbf{n}}^{\mathbf{i}-\mathbf{j}}$ in one case as basis for a reduction, like in the previous section, and in the other one directly, like in $[29,30]$. In other words, what is the difference in representing the fact that $\phi$ is obligatory as $\square_{i}(\neg \phi \rightarrow V)$ and as $\square_{i} \phi$ ? This difference is evident at a semantic level. In $[29,30]$ it is the the set $W_{i}$ (see Section 3.4), which clusters the set of all states in which, we might say, $\neg V$ holds with respect to agent $i$. In our reduction approach, instead, ideal states are clustered inside the set $W_{i}$ by means of the atom $V$. In other words, while in $[29,30]$ contexts are sets of ideal worlds, in our counts-as based reduction contexts just classify what counts as a violation, and it can therefore be possible that violations occur in some of the worlds constituting a context. This difference becomes evident in the representation of violation: if $\mathbf{K D 4} \mathbf{4} \mathbf{n}_{\mathbf{n}}^{\mathbf{i}-\mathbf{j}}$ is directly used as a deontic logic, violations are representable as $\square_{i} \phi \wedge \neg \phi$; while if it is used as basis for a reduction it becomes possible to distinguish between a kind of factual violation $V$, analogous to the previous one, and a kind of local or contextualized violation $\diamond_{i} V$.

\section{A Comparison}

In the formal analysis proposed in [26] a modal logic characterization of countsas statements is also set forth. That approach has a twofold articulation: countsas statements are first formalized by means of a non-normal conditional logic endowed with a minimal model semantics ${ }^{19}$; counts-as statements are then linked to a notion of institutional fact, i.e., a fact holding with respect to an institution or normative system (context) ${ }^{20}$, which is formalized via a $\mathbf{K D}_{\mathbf{n}}$ logic modality $^{21}$. For some reason, the two notions are kept apart from each other and characterized by means of two different logics, which are only in the second instance integrated in one unique framework. This is not the case in our analysis where counts-as itself is, in some sense, an institutional fact, it consisting of a

\footnotetext{
${ }^{19}$ See [11]

${ }^{20}$ See footnote 11 .

${ }^{21}$ For space reasons we refer the reader to [26] for a detailed exposition of those ideas.
} 
subsumption relation which holds with respect to a precise institution (or normative system). Consequently, one modality has been enough for us to provide the desired characterization.

The line of analysis followed in [26] consists in isolating some syntactic constraints of counts-as statements (intuitive axioms and inference rules), and in providing a semantics to the found axiomatization via a minimal model semantics ([11]) which directly mimics those constraints. Given the syntactic flavor of the approach sketched in [26], we will let the consideration of some validities to lead the comparison of the two frameworks.

In order to make the comparison more perspicuous, we are going to use the notation used also in [26]. Therefore, in what follows counts-as conditionals such as $\phi_{1} \Rightarrow_{i} \phi_{2}$ are shortcuts for $\square_{i}\left(\phi_{1} \rightarrow \phi_{2}\right)$; and institutional fact expressions such as $D_{i} \phi$ are shortcuts for $\square_{i} \phi$.

Proposition 4 (Validities)

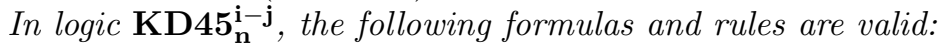

$$
\begin{aligned}
& \phi_{2} \leftrightarrow \psi_{2} /\left(\phi_{1} \Rightarrow_{i} \phi_{2}\right) \leftrightarrow\left(\phi_{1} \Rightarrow_{i} \psi_{2}\right) \\
& \phi_{1} \leftrightarrow \psi_{1} /\left(\phi_{1} \Rightarrow_{i} \phi_{2}\right) \leftrightarrow\left(\psi_{1} \Rightarrow_{i} \phi_{2}\right) \\
& \left(\left(\phi_{1} \Rightarrow_{i} \phi_{2}\right) \wedge\left(\phi_{1} \Rightarrow_{i} \phi_{3}\right)\right) \rightarrow\left(\phi_{1} \Rightarrow_{i}\left(\phi_{2} \wedge \phi_{3}\right)\right) \\
& \left(\left(\phi_{1} \Rightarrow_{i} \phi_{2}\right) \wedge\left(\phi_{3} \Rightarrow_{i} \phi_{2}\right)\right) \rightarrow\left(\left(\phi_{1} \vee \phi_{3}\right) \Rightarrow_{i} \phi_{2}\right) \\
& \left(\phi_{1} \Rightarrow_{i} \phi_{2}\right) \rightarrow\left(\left(\phi_{2} \Rightarrow_{i} \phi_{3}\right) \rightarrow D_{i}\left(\phi_{1} \rightarrow \phi_{3}\right)\right) \\
& \left(\phi_{1} \Rightarrow_{i} \phi_{2}\right) \rightarrow D_{i}\left(\phi_{1} \rightarrow \phi_{2}\right) \\
& \phi \Rightarrow_{i} \phi \\
& \left(\phi_{1} \Rightarrow_{i} \phi_{2}\right) \wedge\left(\phi_{2} \Rightarrow_{i} \phi_{3}\right) \rightarrow\left(\phi_{1} \Rightarrow_{i} \phi_{3}\right) \\
& \left(\phi_{1} \Rightarrow_{i} \phi_{2}\right) \wedge\left(\phi_{2} \Rightarrow_{i} \phi_{1}\right) \rightarrow D_{i}\left(\phi_{1} \leftrightarrow \phi_{2}\right) \\
& D_{i} \phi \leftrightarrow D_{j} D_{i} \phi .
\end{aligned}
$$

Remarkably, our system validates all the intuitive syntactic constraints isolated in [26] (validities 1-6) providing at the same time a semantics grounded on a neat, even if perhaps not definitive, analytical thesis about the nature of countsas rather than on a minimal models one.

Besides, our semantics oriented approach enables the four validities 7-10. While in [26] reflexivity, transitivity, and symmetry of the conditional were explicitly left as open issues, our proposal shows that counts-as conditionals, once they are viewed as conditionals of a classificatory nature, naturally satisfy reflexivity (7), transitivity (8), and a contextualized form of antisymmetry (9). The last validity (10), also not present in [26], is very interesting, and marks a deep difference between the two approaches. The $\mathbf{K D} \mathbf{4 5} \mathbf{n}_{\mathbf{n}}^{\mathbf{i}-\mathbf{j}}$ modality proposed in this work is a $\mathbf{K D}_{\mathbf{n}}$ one extended with two more axioms the meaning of which is to make the nesting of operators reducible ${ }^{22}$. This corresponds to the intuition we already discussed in Section 3.5 according to which what holds in an institution (context) holds irrespectively of the institution (context) from

${ }^{22}$ See the axiomatization exposed in Section 3.4. 
which that fact is considered. Obviously, this cannot be achieved within a $\mathbf{K D}_{\mathbf{n}}$ system that, consequently, appears to be too weak to satisfactorily characterize a notion of institutional fact.

There is also a schema of the framework in [26] which is not valid in ours: $\left(\phi_{1} \Rightarrow_{i} \phi_{2}\right) \rightarrow\left(\phi_{1} \rightarrow D_{i} \phi_{1}\right)$. The idea behind this is that the truth of a countsas conditional should be enough to determine that the truth of the antecedent of the counts-as implies the truth of it within an institution: if something is factually the case, and it determines institutional consequences, then it is also institutionally the case. Though this might seem to some extent reasonable, it is no surprise that our semantics does not validate it. In fact, it implies that $\left(\phi_{1} \Rightarrow_{i} \phi_{2}\right) \rightarrow\left(\neg D_{i} \phi_{1} \rightarrow \neg \phi_{1}\right)$, which is manifestly too strong: if I am not officially able to drive a car, this does not imply that I am not able to drive a car since I might be a formula 1 pilot without a driving license. Nevertheless, rule $\left(\phi_{1} \Rightarrow_{i} \phi_{2}\right) \wedge \phi_{1} / D_{i} \phi_{1}$, which does not give rise to any contrapositive counterintuitive formula, is still derivable in our framework (trivially, because of the necessitation rule).

Finally, the issue concerning what in [26] is called transfer problem deserves also some words since it marks another important difference between the two proposals. This problem can be exemplified as follows: suppose that somebody brings it about that a priest effects a marriage, does this count as the creation of a state of marriage? In other words, is the possibility to constitute a marriage transferable to anybody who brings it about that the priest effects the ceremony? Technically, can the antecedent of counts-as be strengthened? In [26] the answer is negative, because only the performance of the ceremony by a priest can effectively count as the creation of a marriage state, and the formalism is indeed geared toward avoiding the transfer problem. Here instead, the analytical option of viewing counts-as as contextual classification commits us to answer affirmatively to the previous question. In fact, our formalization of counts-as does exhibit the transfer problem (strengthening of the antecedent holds), but this is exactly what it would be intuitively expected: whatever situation in which a priest performs a marriage ceremony is classified as a situation in which a marriage state comes to be (with respect to the relevant institutional context).

\section{Discussion and Conclusions}

To conclude, our contribution consists in a multi-modal logic formalization of counts-as statements interpreted as contextual classificatory statements.

In the last part of the work, we also provided a close formal comparison of our proposal with the similar approach exposed in [26], discussing analytic and technical differences. We show how our semantics-driven approach appears to be more suitable in capturing many intuitive features of counts-as statements, providing also clear insights in what they actually mean.

There are some issues related to the investigations presented in these pages.

- The modal logic account we provided suffers of the usual shortcomings ling 
in the classical logic paradigm such as the $\square_{i}$ necessitation of tautologies, which also means that " $T$ counts-as $T$ " in context $i$, in symbols, $\square_{i}(\top \rightarrow$ T) and alike.

- Non-monotonicity issues such as the ones risen in [16] are completely disregarded. On the other hand, our monotonic counts-as can straightforwardly be merged in a suitable argumentation system ([34]) providing the desired defeasible properties. An alternative solution would be to handle non-monotonic aspects of counts-as via monotonic techniques, importing the ideas from the epistemic default logic developed in [31] which, as we saw in Section 4.1, makes use of essentially the same logical machinery. However, the point is also theoretical: is counts-as constitutively defeasible? Our answer is that, if it is interpreted as contextual classification, it is not: classification is monotonic and contexts just localize it allowing therefore for multiple inconsistent classifications (See Section 2). We deem defeasibility to be a phenomenon arising at the top of contextuality once assertions are kept context-independent and inconsistencies are handled via an explicit notion of exception.

- The proposal is based on a precise understanding of the counts-as phenomenon, namely "counts-as means contextual classification". This definitely makes our approach not completely exhaustive in the understanding of the whole institutional phenomenon of counts-as, but it has the unquestionable advantage of clarifying a precise analytical option, framing it in a rigorous setting. In particular, what a classificatory view of counts-as implies is somehow the absence of a satisfactory explanation of the constitutive character of counts-as, of its bringing something new, something constituted, about, of its generating something: classification is something flat and somehow exogenous, while counts-as seems to incorporate also a kind of generative dimension ${ }^{23}$. In fact, schemata such as (7) in Section 5 would result counterintuitive in the light of a formalization of the generative character of counts-as statements.

- The framework does not deal, at the present stage, with counts-as statements concerning actions and activities such as: "signing the copyright form counts as consenting to transfer the copyright". We think that dynamics can nevertheless be added to our framework via appropriate forms of dynamic logic ${ }^{24}$. The introduction of dynamic logic would also provide the possibility to account for kinds of constitutive actions which create contexts ("context creation") and which modify their content ("context modification").

- An aspect completely disregarded is also the terminological independence which characterizes different institutions or normative systems we stressed in [19], and which constitutes a common denominator of various work on

\footnotetext{
${ }^{23}$ Some of these aspects are informally discussed in [39].

${ }^{24}$ See [6].
} 
context theory ${ }^{25}$. Each normative system should have its own language to talk about the properties relevant for itself: public park regulations do not talk about immigrants. This constitutes of course a problematic aspect to be treated within a standard modal logic setting like ours, where one unique language is presupposed and all atoms receive an evaluation in each context.

These issues deserve all particular attention in order to deepen and enrich the analysis presented here. They mark the directions for future work.

\section{References}

[1] C. E. Alchourrón and E. Bulygin. Normative Systems. Springer Verlag, Wien, 1986.

[2] A. Anderson. The formal analysis of normative concepts. American Sociological Review, 22:9-17, 1957.

[3] A. Anderson. A reduction of deontic logic to alethic modal logic. Mind, 22:100-103, 1958.

[4] F. Baader, D. Calvanese, D. McGuinness, D. Nardi, and P. Patel-Schneider. The Description Logic Handbook. Cambridge University Press, Cambridge, 2002.

[5] M. Benerecetti, P. Bouquet, and C. Ghidini. Contextual reasoning distilled. Journal of Experimental and Theoretical Artificial Intelligence (JETAI), 12(3):279-305, 2000.

[6] P. Blackburn, M. de Rijke, and Y. Venema. Modal Logic. Cambridge University Press, Cambridge, 2001.

[7] G. Boella and L. Van der Torre. Regulative and constitutive norms in normative multiagent systems. In Proceedings of KR2004, Whistler, Canada, pages 255-266, 2004.

[8] E. Bulygin. On norms of competence. Law and Philosophy 11, pages 201$216,1992$.

[9] S. Buvač, S. V. Buvač, and I. A. Mason. The semantics of propositional contexts. Proceedings of the eight ISMIS. LNAI-869, pages 468-477, 1994.

[10] S. V. Buvač and I. A. Mason. Propositional logic of context. Proceedings AAAI'93, pages 412-419, 1993.

[11] B. F. Chellas. Modal Logic. An Introduction. Cambridge University Press, Cambridge, 1980.

${ }^{25}$ See for example [5]. 
[12] P. d'Altan, J.-J. C. Meyer, and R. Wieringa. An integrated framework for ought-to-be and ought-to-do constraints. In Y. H. Tan, editor, Working Papers of the Workshop om Deontic and Non-Monotonic Logics, pages 1445, Rotterdam, 1993.

[13] R. Fagin, J. Halpern, and M. Vardi. Belief, awareness and limited reasoning. Artificial Intelligence, 34:39-76, 1988.

[14] D. Gabbay, A. Kurucz, F. Wolter, and M. Zakharyaschev. Manydimensional modal logics. Theory and applications. Elsevier, 2003.

[15] A. I. Goldman. A Theory of Human Action. Princeton University Press, Princeton, 1976.

[16] G. Governatori, J. Gelati, A. Rotolo, and G. Sartor. Actions, institutions, powers. preliminary notes. In International Workshop on Regulated AgentBased Social Systems: Theories and Applications(RASTA'02), pages 131$147,2002$.

[17] D. Grossi, H. Aldewereld, J. Vázquez-Salceda, and F. Dignum. Ontological aspects of the implementation of norms in agent-based electronic institutions. Presented at NorMAS, 2005.

[18] D. Grossi and F. Dignum. From abstract to concrete norms in agent institutions. Proceedings of FAABS III Workshop, LNAI 3228, Washington, April 2004.

[19] D. Grossi, F. Dignum, and J.-J. C. Meyer. Contextual taxonomies. In J. Leite and P. Toroni, editors, Proceedings of CLIMA V Workshop, Lisbon, September, pages 2-17, 2004.

[20] J. Hage and B. Verheij. The law as a dynamic interconnected system of states of affairs. IJHCS: International Journal of Human-Computer Studies, 51:1043-1077, 1999.

[21] H. L. A. Hart. Positivism and the separation of law and morality. Harvard Law Review, 71:593-629, 1958.

[22] H. L. A. Hart. The Concept of Law. Clarendon Press, Oxford, 1961.

[23] J. Hintikka. Knowledge and Belief: An Introduction to the Logic of the Two Notions. Cornell University Press, Ithaca, 1962.

[24] A. J. I. Jones and M. Sergot. Deontic logic in the representation of law: towards a methodology. Artificial Intelligence and Law 1, 1992.

[25] A. J. I. Jones and M. Sergot. On the characterization of law and computer systems. Deontic Logic in Computer Science, pages 275-307, 1993.

[26] A. J. I. Jones and M. Sergot. A formal characterization of institutionalised power. Journal of the IGPL, 3:429-445, 1996. 
[27] J. Krabbendam and J.-J. C. Meyer. Contextual deontic logics. In P. McNamara and H. Prakken, editors, Norms, Logics and Information Systems, pages 347-362, Amsterdam, 2003. IOS Press.

[28] A. Lomuscio and D. Nute, editors. Proceedings of DEON'04, 2004.

[29] A. Lomuscio and M. Sergot. Investigations in grounded semantics for multiagent systems specification via deontic logic. Technical report, Imperial College, 2000.

[30] A. Lomuscio and M. Sergot. Deontic intepreted systems. Studia Logica 75, pages 63-92, 2003.

[31] J. Meyer and W. van der Hoek. Epistemic Logic for AI and Computer Science, volume 41 of Cambridge Tracts in Theoretical Computer Science. Cambridge University Press, 1995.

[32] P. Nayak. Representing multiple theories. In Proceedings of the 12th National Conference on Artificial Intelligence, Volume 2. Seattle, WA, USA, pages 1154-1160. AAAI Press, 1994.

[33] A. Peczenik. On Law and Reason. Kluwer, Dordrecht, 1989.

[34] H. Prakken and G. Vreeswijk. Logics for defeasible argumentation. Handbook of Philosophical Logic vol. IV, pages 218-319, 2002.

[35] F. Raimondi and L. Lomuscio. Automatic verification of deontic properties of multi-agent systems. In A. Lomuscio and D. Nute, editors, Proceedings of DEON'04, pages 228-242, 2004.

[36] A. Ross. Directives and Norms. Routledge \& Kegan Paul, London, 1968.

[37] D. W. P. Ruiter. A basic classification of legal institutions. Ratio Juris, 10:357-371, 1997.

[38] J. Searle. Speech Acts. Cambridge University Press, Cambridge, 1986.

[39] J. Searle. The Construction of Social Reality. Free Press, 1995.

[40] R. Stalnaker. On the representation of context. In Journal of Logic, Language, and Information 7, pages 3-19. Kluwer, 1993.

[41] G. H. Von Wright. Norm and Action. A Logical Inquiry. Routledge, London, 1963. 